\title{
DERIVATIONS AND GENERATIONS OF FINITE EXTENSIONS
}

\author{
BY CARL FAITH ${ }^{1}$
}

Communicated by Nathan Jacobson, May 8, 1961

Let $k$ be a given ground field, let $\mathcal{F}_{r}$ denote the class of finite (= finitely generated) field extensions of $k$ of tr.d. ( $=$ transcendence degree) $\leqq r$, and let $\boldsymbol{n}$ be the function defined on $\mathcal{F}=\cup_{0}^{\infty} \mathcal{F}_{\boldsymbol{r}}$ by: for any $L \in \mathcal{F}, \boldsymbol{n}(L)=$ the minimal number of generators of $L / k$. Classically it is known for suitable $k$ that there exist purely transcendental extensions $L / k$ having tr.d. 2 , and containing impure subextensions of tr.d. 2, a fact which shows that in general $\boldsymbol{n}$ is not monotone in $\mathcal{F}$ for all $k$. The main result of this note establishes that these "counterexamples to Lüroth's theorem" constitute the only barriers to the monotonicity of $\boldsymbol{n}$ (see Theorem 2 for a precise statement). In particular it is demonstrated that $\boldsymbol{n}$ is montone on $\mathcal{F}_{1}$ for arbitrary $k$, a result which appears new even when restricted to the subclass $\mathfrak{F}_{0}$ of finite algebraic extensions of $k$.

A result of independent (and possibly more general) interest, which is proved below, and which is essential to our proof of the statements above, is that $\operatorname{dim} D$ is montone on $\mathcal{F}$, where for any $L \in \mathcal{F}, D(L)$ is the vector space over $L$ of $k$-derivations of $L$. The connection between $\boldsymbol{n}$ and $\operatorname{dim} D$ is given in the lemma.

Lemma. Let $L / k$ be a finite extension of tr.d. $r$, let $s=\operatorname{dim} D(L)$, and let $n=n(L)$. Then $s \leqq n \leqq s+1$; if $s>r$, then $n=s .{ }^{2}$

Proof. It is known (e.g. [3, Theorem 41, p. 127]) that $s$ is the smallest natural number ${ }^{3}$ such that there exist elements $u_{1}, \cdots, u_{s} \in L$ such that $L$ is separably algebraic over the field $U=k\left(u_{1}, \cdots, u_{s}\right)$. Then $L=U(a)$ for some $a \in L$, so that $s \leqq n \leqq s+1$.

If $s>r$, there exists $u_{q}$ in the set $S=\left\{u_{1}, \cdots, u_{s}\right\}$ such that $u_{q}$ is algebraically dependent over $k$ on the complement of $u_{q}$ in $S$. For convenience renumber so that $u_{\theta}$ is algebraic $c^{4}$ over the field $T=k\left(u_{1}, \cdots, u_{s-1}\right)$. A short argument shows that $L=U(a)$ for some

1 National Science Foundation Postdoctoral Fellow in the Institute for Advanced Study, on leave from Pennsylvania State University.

2 Expressed in the other words: If $L / k$ is not separably generated, then $n(L)$ $=\operatorname{dim} \mathscr{D}(L)$.

${ }^{3}$ Strictly speaking the notation should allow for the case $s=0$. By agreement then $U=k$.

In case $s=1$ set $T=k$. 
$a \in L$ which is separably algebraic over $T$. Thus $L=T\left(u_{s}, a\right)$ is generated over $T$ by two elements one of which is separable over $T$. Then, by a classic result in field theory (cf. $\left[2, \S 43\right.$, p. 138]), $L=T\left(u_{s}^{\prime}\right)$ for suitable $u_{s}^{\prime} \in L$. Clearly then $n=s$. Q.E.D.

If $L / k$ is a finite extension, and $L^{\prime} / k$ a subextension, in general not every derivation of $D\left(L^{\prime}\right)$ can be extended to a derivation in $D(L)$. Nevertheless, the theorem below shows that $\operatorname{dim} D$ is a monotone function.

THEOREM 1. Let $L / k$ be a finite field extension, and let $L^{\prime} / k$ be any subextension. Then $s=\operatorname{dim} D(L) \geqq s^{\prime}=\operatorname{dim} D\left(L^{\prime}\right)$.

PRoof. Let $r=\operatorname{tr}$.d. $L / k$ and $r^{\prime}=\operatorname{tr}$.d. $L^{\prime} / k$. It is easy to see that it suffices to consider only the case $r=r^{\prime}$. For if $r^{\prime}<r$, then there exists a field extension $L^{\prime \prime}$ contained in $L$ which is purely transcendental over $L^{\prime}$ and such that tr.d. $L^{\prime \prime} / k=r$. Since $L^{\prime \prime} / L^{\prime}$ is a pure extension, every $D^{\prime} \in \mathbb{D}\left(L^{\prime}\right)$ is extendable to a derivation $D^{\prime \prime}$ in $\mathscr{D}\left(L^{\prime \prime}\right)$. It is an easy exercise to show that if $D_{1}^{\prime}, \cdots, D_{t}^{\prime}$ are linearly independent over $L^{\prime}$, then $D_{1}^{\prime \prime}, \ldots, D_{t}^{\prime \prime}$ are linearly independent over $L^{\prime \prime}$, so that $\operatorname{dim} \mathscr{D}\left(L^{\prime \prime}\right) \geqq s^{\prime}$. Hence it remains only to show that $s \geqq s^{\prime}$ when $r=r^{\prime}$. An argument similar to the one just completed shows that $s \geqq s^{\prime}$ when $L / L^{\prime}$ is separable. The proof now proceeds by induction on the algebraic degree $\left|L: L^{\prime}\right|$ of the extension $L / L^{\prime}$. One can therefore assume the theorem for all extensions $L^{\prime \prime}$ of $k$ which contain $L^{\prime}$ and such that $\left|L^{\prime \prime}: L^{\prime}\right|\left\langle\left|L: L^{\prime}\right|\right.$. Then clearly one can suppose that $L^{\prime}$ is a maximal proper subfield of $L$. Since the separable case already has been decided, assume that $k$ has characteristic $p>0$, and that $L / L^{\prime}$ is inseparable. Then the maximality of $L^{\prime}$ shows that $k\left(L^{p}\right) \subseteq L^{\prime}$. By $[1$, p. 218] or [3, Theorem 41, p. 127], one has

$$
p^{s}=\left|L: k\left(L^{p}\right)\right|, \text { and } p^{s^{\prime}}=\left|L^{\prime}: k\left(L^{\prime p}\right)\right|,
$$

so that the inclusions

$$
L \supset L^{\prime} \supseteq k\left(L^{p}\right) \supseteq k\left(L^{\prime p}\right)
$$

together with the inequality

$$
\left|L: L^{\prime}\right| \geqq\left|k\left(L^{p}\right): k\left(L^{\prime p}\right)\right|
$$

yield the desired inequality $p^{8} \geqq p^{8^{\prime}}$, that is, $s \geqq s^{\prime}$. Q.E.D.

CoROLlary. Let $L / k$ be a finite extension, and let $L^{\prime} / k$ be any subextension. Then, if either $L / k$ or $L^{\prime} / k$ is not separably generated, then $\boldsymbol{n}(L) \geqq n\left(L^{\prime}\right)$. 
Proof. Let $\mathbf{s}=\operatorname{dim} D(L), \boldsymbol{r}=\operatorname{tr} . \mathrm{d} . L / k, \boldsymbol{n}=\mathbf{n}(L)$, and let $\boldsymbol{s}^{\prime}, \boldsymbol{r}^{\prime}$, and $n^{\prime}$ be the corresponding integers for $L^{\prime} / k$. If $L^{\prime} / k$ is not separably generated, neither is $L / k$, so that we can assume that $L / k$ is not separably generated in either case, that is, that $s \geqq r+1$. Then $n=s$ by the lemma, whence $n=s \geqq s^{\prime}$ by the theorem. If $n^{\prime}=s^{\prime}$, we are through, and if $n^{\prime} \neq s^{\prime}$, then $n^{\prime}=s^{\prime}+1=r^{\prime}+1$ by the lemma again. Since $\boldsymbol{r} \geqq \boldsymbol{r}^{\prime}$, this latter equality yields

$$
n=s \geqq r+1 \geqq r^{\prime}+1=s^{\prime}+1=n^{\prime},
$$

as desired.

The corollary is surprising in view of the troublesomeness usually associated with nonseparably generated extensions.

Before stating the last result, it is convenient to make the definition: $k$ is an (r)-field in case no pure transcendental extension of $k$ of tr.d. $r$ contains an impure subextension of tr.d. $r$ over $k$. Clearly if $\boldsymbol{n}$ is monotone in $\mathcal{F}_{r}$, then $k$ must be an $(\boldsymbol{m})$-field, $\boldsymbol{m}=0,1, \cdots, \boldsymbol{r}$. Our next theorem establishes the converse.

THEOREM 2. If $k$ is an (r)-field, and if $L / k$ is a finite extension of tr.d. $\boldsymbol{r}$, then $\boldsymbol{n}=\boldsymbol{n}(L) \geqq \boldsymbol{n}^{\prime}=\boldsymbol{n}\left(L^{\prime}\right)$ for any subextension $L^{\prime} / k$ of $L / k$.

Proof. Let $s, r, n$, and their primes be defined as in the corollary. If $\boldsymbol{s}^{\prime}>\boldsymbol{r}^{\prime}$, then $\boldsymbol{n}>\boldsymbol{n}^{\prime}$ by the corollary. If $L^{\prime} / k$ is purely transcendental, then trivially $n \geqq n^{\prime}$. Otherwise $\boldsymbol{s}^{\prime}=\boldsymbol{r}^{\prime}$ implies by the lemma that $\boldsymbol{n}^{\prime}=\boldsymbol{s}^{\prime}+1=\boldsymbol{r}^{\prime}+1$. Then, since $k$ is an $(\boldsymbol{r})$-field, necessarily $\boldsymbol{n} \geqq \boldsymbol{r}+1$ $=\boldsymbol{r}^{\prime}+1=\boldsymbol{n}^{\prime}$, if $\boldsymbol{r}=\boldsymbol{r}^{\prime}$. If $\boldsymbol{r}>\boldsymbol{r}^{\prime}$, then clearly $n \geqq r \geqq \boldsymbol{r}^{\prime}+1=\boldsymbol{n}^{\prime}$. Q.E.D.

By definition, any field is a (0)-field, and, by Lüroth's theorem, any field is a (1)-field. Thus, the theorem implies the corollary:

Corollary. Let $k$ be an arbitrary field. Then $n$ is monotone in the class $\mathfrak{F}_{1}$ of finite extensions of $t r . d$. $\leqq 1$ over $k$; in particular, $n$ is monotone in the class $\mathcal{F}_{0}$ of finite algebraic extensions of $k$.

A consequence of Theorem 2 and the theorem of CastelnuovoZariski (see [4]) is the following:

COROLlARy. Let $k$ be an algebraically closed field of characteristic 0. Then $n$ is monotone in the class $F_{2}$ of finite extensions of $t r . d . \leqq 2$ over $k$.

A possible value of Theorem 2 is that in order to show that a given field is not an (r)-field, it is possible to do this by showing that $n$ is not monotone on its finite extensions of tr.d. $r$, that is, one need not restrict one's attention to the pure transcendental extensions of $k$. 


\section{REFERENCES}

1. Nathan Jacobson, Abstract derivations and Lie algebras, Trans. Amer. Math. Soc. vol. 42 (1937) pp. 206-224.

2. B. L. van der Waerden, Algebra I, Vierte Auflage, Berlin-Göttingen-Heidelberg, 1955.

3. Oscar Zariski and Pierre Samuel, Commutative algebra, vol. I, Princeton, 1958.

4. Oscar Zariski, On Castelnuovo's criterion of rationality $p_{a}=P_{2}=0$ of an algebraic surface, Illinois J. Math. vol. 2 (1958) pp. 303-315.

INSTITUTE FOR AdVANCEd STUdy 